\title{
Functional Emesis
}

\author{
Stefan L. Popa ${ }^{1}$, Giuseppe Chiarioni ${ }^{2}$, Liliana David ${ }^{1}$, George I. Golea ${ }^{1}$, Dan L. Dumitrascu ${ }^{1}$
}

1) $2^{\text {nd }}$ Medical Department,

Iuliu Hațieganu University of Medicine and Pharmacy,

Cluj-Napoca, Romania

2) Division of

Gastroenterology of the

University of Verona, AOUI

Verona, Italy and Division

of Gastroenterology and

Hepatology \& Center for

Functional GI and Motility

Disorders, University of

North Carolina at Chapel Hill,

Chapel Hill, NC, USA
Address for correspondence:

Liliana David, $\mathrm{PhD}$

$2^{\text {nd }}$ Medical Department

Clinicilor Street, No. 3-5,

RO 400003 Cluj-Napoca,

Romania

lilidavid2007@yahoo.com

Received: 27.06.2019

Accepted: 27.08.2019

\begin{abstract}
Emesis is an essential survival response, because consumption of food is a key element in the survival behavior and the act of feeding can expose the majority of internal organs to toxins, altered food, viruses, bacteria or fungi. The relationship between visceral or vestibular stimuli and the anatomical structures of the brain-gut axis explain the modulation of the gastrointestinal response. We aimed to review comprehensively the current knowledge on emesis occurring in the disorders of brain-gut interaction (DBGI), i.e. functional conditions presenting with emesis. PubMed, Cochrane Library, EMBASE and WILEY databases were screened for relevant publications from the last 20 years regarding brain-gut disorders manifesting emesis. We included in the search also idiopathic gastroparesis, as a condition paralleling the postprandial distress syndrome. We critically analyzed 2 articles about chronic nausea and vomiting syndrome, 9 articles about cyclic vomiting syndrome, 9 articles about cannabinoid hyperemesis, 10 articles about idiopathic gastroparesis and 6 articles about gastroparesis like-syndrome. Contradictory results regarding the implication of environmental factors in the development of emesis in DBGI demonstrate that the underlying pathogenesis is still not completely understood.
\end{abstract}

Key words: emesis - vomiting - functional gastrointestinal disorders - disorders of brain-gut interaction.

Abbreviations: CHS: cannabinoid hyperemesis syndrome; CNVS: chronic nausea and vomiting syndrome; CVS: cyclic vomiting syndrome; DBGI: disorders of brain-gut interaction; ECS: endogenous cannabinoid system; FGID: functional gastrointestinal disorders; GES: gastric electrical stimulation; GP: gastroparesis; GLS: gastroparesis-like syndrome; ICC: interstitial cells of Cajal; IGP: idiopathic gastroparesis tGES: temporary gastric electrical stimulation; TSS: total symptom score.

\section{INTRODUCTION}

Emesis is a reflex which integrates a complex set of actions of the inspiratory and expiratory muscles in order to produce an involuntary, forceful expulsion of the contents of stomach through the mouth and rarely through the nose [1]. Emesis must be distinguished from regurgitation and rumination.

The first description of emesis as a "reflex arc" dates back to more than four centuries ago by the French philosopher Descartes. Charles Sherrington, an English physiologist, conducted complex experiments on spinal cord reflexes in the early years of the XX century, demonstrating the link between the central nervous system, peripheral nervous system, vestibular function and emesis [2].

Emesis is an essential survival response, because consumption of food is a key element in the survival behavior and the act of feeding can expose the majority of internal organs to toxins, altered food, viruses, bacteria and fungi. Similar with the survival response of emesis, diarrhea has the role of removing toxins and pathological agents from the lower intestines.

The activation of the emesis reflex arc has the function of modulating the gastrointestinal function, the response to stress environmental changes, the regulation of the gastrointestinal immune system, mucosal inflammation and intestinal microbiota (Fig.1) [3-8].

Functional gastrointestinal disorders (FGID) are disorders of the digestive system in which symptoms cannot be explained by the presence of structural or tissue abnormality.

Disorders of Brain-Gut Interactions (DBGI) is the new name of FGID and the diagnosis is mainly clinical [1-3]. Emesis 


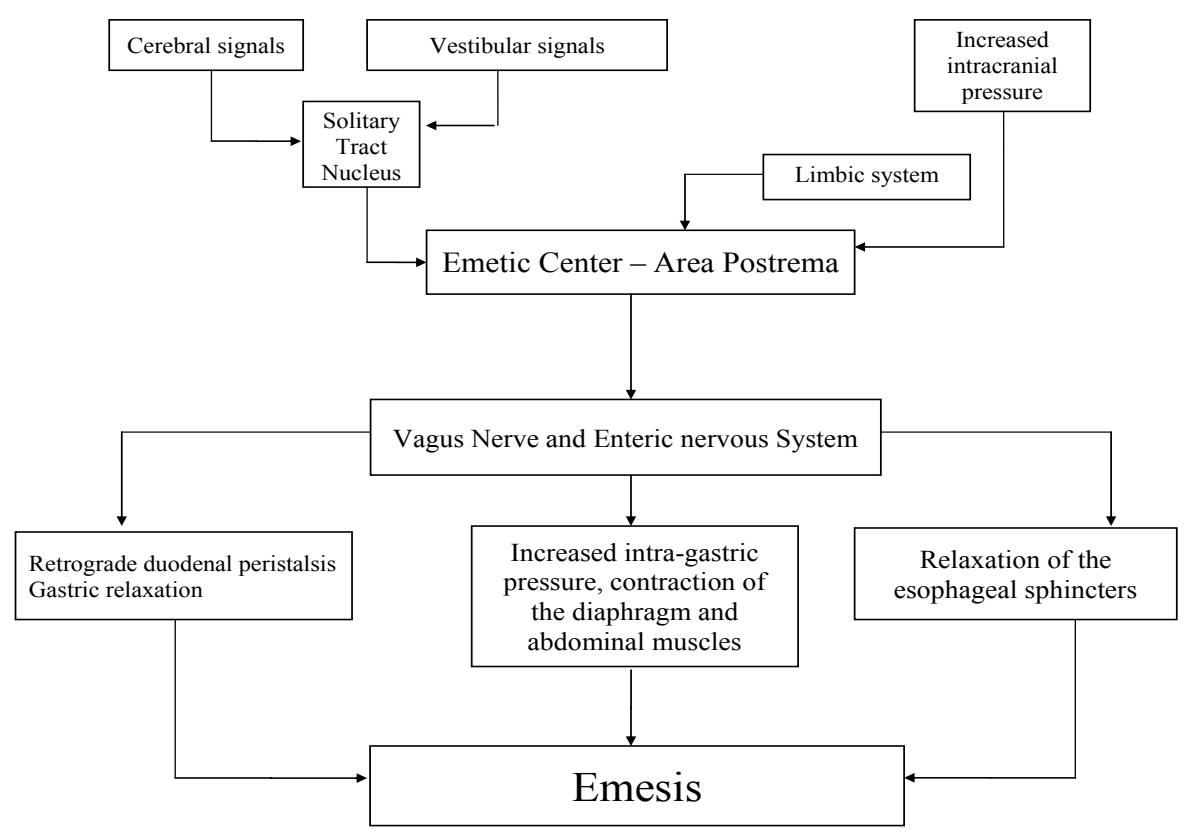

Fig. 1. The pathogenesis of emesis.

is encountered in some of these DBGI. The diagnosis of these disorders relies on the symptom-based Rome IV Criteria [3]. According to these, the DBGI presenting vomiting are included in the Gastroduodenal DBGI. They are classified in three syndromes: chronic nausea vomiting syndrome (CNVS), cyclic vomiting syndrome (CVS), cannabinoid hyperemesis syndrome (CHS) [3]. Beside this, emesis may occur accompanying nausea in postprandial distress syndrome, one of the subtypes of functional dyspepsia.

Three years have elapsed since the issue of the Rome IV Criteria and we searched for an update review on the current knowledge of the DBGI manifested with emesis. We also looked for current evidence on idiopathic gastroparesis (IGP), which is a parallel disorder to functional dyspepsia. We report additionally on the emerging entity of the gastroparesis-like syndrome (GLS).

PubMed, Cochrane Library, EMBASE and WILEY databases were screened for relevant publications regarding brain-gut disorders manifested with emesis between January 1999 and January 2019. The search terms included: (emesis OR vomiting OR vomit) AND (brain-gut OR brain OR functional gastrointestinal disorders OR FGID OR chronic nausea) AND (vomiting syndrome OR cyclic vomiting syndrome OR cannabinoid hyperemesis OR idiopathic gastroparesis OR gastroparesis like-syndrome). Exclusion criteria were: studies written in languages other than English, studies not performed in humans, abstracts, conference presentations, letters to the editor, editorials, comments and opinions and these were not taken into consideration. Articles treating the subject of rumination syndrome were also excluded. The articles included in this review refer only to DBGI that include the brain-gut axis pathology and emesis as the predominant symptom.

The initial search identified 1898 articles, out of which 257 potentially appropriate articles were reviewed. Of these 257 articles, 36 articles were retained for this review (Fig. 2). Two articles analyzed CNVS, 9 articles analyzed CVS, 9 articles analyzed cannabinoid hyperemesis, 10 articles analyzed IGP and 6 articles analyzed GLS.

\section{Chronic nausea and vomiting syndrome}

According to the Rome IV Diagnostic Criteria, all the following clinical and lab criteria must be fulfilled for the last 3 months with symptom onset at least 6 months prior to diagnosis of CNVS: bothersome nausea (severe enough to impact on usual activities), occurring at least 1 day per week and/or one or more episodes of emesis per week. It should be differentiated from self-induced vomiting, eating disorders, regurgitation or rumination. No evidence of organic, systemic or metabolic diseases should be found on routine investigations including upper endoscopy [9].

The prevalence of CNVS is unknown, although nausea is reported at least weekly by $3 \%$ of normal population [9].

The treatment options include antiemetic agents divided into 5 classes: 1) histamine $\mathrm{H} 1$ antagonists (meclizine, promethazine); 2) muscarinic M1 antagonists (scopolamine); 3) dopamine D2 antagonists (prochlorperazine); 4) serotonin 5-HT3 antagonists (ondansetron, granisetron), neurokinin NK1 antagonists (aprepitant); 5) cannabinoids (dronabinol) $[3,9,10]$. Clinical trials and therapeutic guidelines are lacking.

\section{Cyclic vomiting syndrome}

Cyclic vomiting syndrome was first described in children by Samuel Gee in 1882 and is a chronic functional gastrointestinal disorder of unknown etiology that is characterized by recurrent and episodes of rapid-fire vomiting between varying periods of completely normal health. The on-and-off stereotypical pattern of paroxystic vomiting is pathognomonic [11].

The Rome IV diagnostic criteria for CVS include of the following criteria: (1) at least 2 periods of unremitting vomiting with or without retching, lasting hours to days within a 6-month period; (2) a return to the usual state of health that lasts weeks to months [12]. 


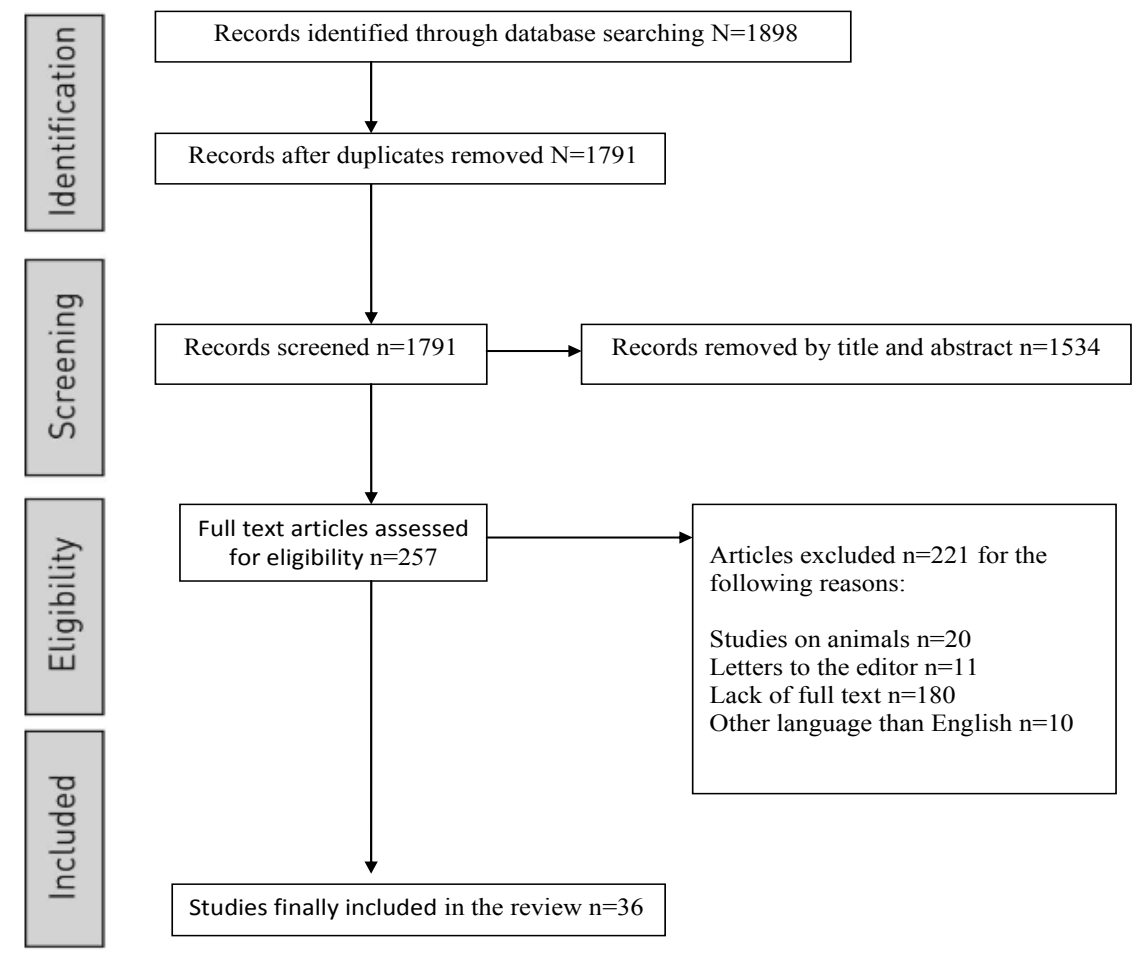

Fig. 2. Flow diagram for study selection.

The differential diagnostic between CNVS and CVS is made using temporal criteria, because CVS was described to have four temporal phases: 1) a pre-emetic period with nausea, pallor, diaphoresis; 2) intense emesis up to 30 episodes daily, often with associated epigastric or diffuse abdominal pain and/or diarrhea; 3 ) a recovery phase with gradual symptom resolution of nausea and vomiting; 4) interepisodic period without vomiting [9].

The true incidence and prevalence of CVS are unknown. Precise data about the epidemiology of CVS are available only for the population in Australia, where Cullen and MacDonald estimated the prevalence of CVS at $2.3 \%$ [13]. Initially, psychosocial factors were attributed to the pathogenesis of CVS, but the recent data show that mitochondrial DNA (mtDNA) mutations may be involved in the pathogenesis of CVS $[14,15]$. Furthermore, $86 \%$ of children with CVS and neuromuscular disease had a history of migraines on the matrilineal side and two mtDNA polymorphisms (16519T and $3010 \mathrm{~A}$ ) are expressed with a high degree of frequency [14-16]. A study that included a small number of patients, found that adult-onset of CVS, unlike pediatric onset of CVS, was not associated with these polymorphisms, suggesting a degree of genetic distinction [16].

A study on 31 patients with CVS by Hikita et al. [17] showed that the median duration of the disease was 66 months and that $44 \%$ of the patients seen for follow-up, developed a severe migraine. The study also showed high serum levels of adrenocorticotropic hormone and antidiuretic hormone in patients with CVS. Furthermore significant correlations between attack duration and antidiuretic hormone and adrenocorticotropic hormone levels were described [17].

In conclusion, the pathogenesis of CVS is multifactorial. A major pitfall in the current medical literature describing CVS is represented by the low number of subjects included in the majority of studies. Further studies are required to discover the exact mechanisms underlying the pathogenesis of this disorder.

Although patients are symptomatic only $10 \%$ of the time, CVS has a major economic impact and it is medically and socially disabling. More than a half of patients require intravenous (IV) fluids and the average annual cost of testing, treatment, and absenteeism is high. In adults, substantial morbidity is associated with CVS, perhaps because of lack of awareness and resultant delays in diagnosis. A study on 41 patients and 293 procedures performed by Fleisher et al. showed that 17 surgical procedures, including 10 cholecystectomies, appendectomies, laparotomies, pyloroplasties and a hysterectomy, were performed without any therapeutic benefit $[18,19]$. Adults and children with CVS also have multiple emergency department consultations and day offs and the diagnosis is frequently mistaken [19]. Sleep deprivation has been described by Fleisher et al. as an important trigger for patients with CVS and the same author has described a $70 \%$ decrease in frequency of episodes only with lifestyle changes without pharmacological, psychological or surgical therapy $[18,19]$. Contrary, a study performed by Li et al. [11] demonstrated that avoiding dietary triggers such as chocolate, cheese, and monosodium glutamate can prevent episodes without the use of drugs, emphasizing the role of diet in the pathogenesis and outcome of FGID.

\section{Cannabinoid hyperemesis syndrome}

Cannabis is the most frequently used illicit drug in the world, with more than 160 million users worldwide $[20,21]$. In the United States, there are 2.4 million new users every year. The number of cannabis user has increased dramatically 
as a consequence of the fast changing political landscape and laws that permit recreational medicinal use of cannabis [21].

Cannabinoid hyperemesis syndrome is characterized by significant nausea, vomiting, diffuse abdominal pain, and relief with hot showers [22]. The most important diagnostic criteria is chronic regular cannabis use. Furthermore, resolution of symptoms appears after stopping cannabis. Compulsive hot baths or showers are associated with symptom relief [22].

Patients follow an extensive evaluation in order to establish a correct diagnosis; frequently they perform unnecessary procedures including advanced imaging, endoscopy, expensive laboratory tests and repeatedly emergency department visits. The diagnosis is frequently mistaken and a delay in finding an appropriate treatment appears. The consequence is an increasing economic burden with no improvement of treatment. Complications of CHS include hospitalizations for severe arrhythmias, dehydration and electrolyte imbalance, ketonuria, mild leukocytosis [23]. If patients deny cannabis use or other illicit drugs but a clinical suspicion remains, a urine drug screen is recommended. Imagistic methods are not recommended because there are no specific radiological findings suggestive of the diagnosis.

Dysregulation of the endogenous cannabinoid system (ECS) is a physiologic regulator of gut motility and is involved in the reflex of emesis and is described as an important pathogenic mechanism in CHS [24-28].

Endogenous cannabinoid system is responsible for the complex effects of cannabis and includes the following components: ligands $\mathrm{N}$-arachidonoylethanolamine (anandamide) and 2-arachidonoylglycerol (2-AG), their biosynthetic and degradative enzymes, and the cannabinoid receptors $\mathrm{CB} 1$ and $\mathrm{CB} 2[8,24,27,28]$. Other pathogenic mechanisms include accumulation of cannabinoid metabolites in the brain and fatty tissues inducing a toxic effect, desensitization and downregulation of $\mathrm{CB} 1$ receptors causing rebound vomiting and spasmodic pain and paradoxical and plastic changes in expression and downstream effects of cannabinoid receptors [8, 27, 28].

Patients presenting with emesis after a period of chronic cannabis use may have a genetic variation in their hepatic enzymes that results in an increase of the levels of cannabis metabolites, which explains the symptomatology [8, 24, 28].

If the cannabis administration is stopped, in most cases symptoms disappear. If the patients are resistant to the indication of cannabinoid abstinence, referral to an appropriate psychiatric dependency department is recommended.

\section{Idiopathic gastroparesis}

Gastroparesis (GP) is a chronic motility disorder characterized by delayed gastric emptying in the absence of organic modifications leading to mechanical obstruction [29]. It is frequently present in patients with diabetes, Parkinsonism and abdominal surgery affecting the vagus nerve. Rare causes include: mononucleosis, scleroderma, EhlersDanlos syndrome, chronic renal failure, hypochlorhydria, intestinal pseudoobstruction, eating disorders, mitochondrial disease, narcotic analgesic or anticholinergic medications, amyloidosis, heavy cigarette smoking, mesenteric ischemia and paraneoplastic syndrome [30-34].
Gastroparesis was first described among patients with diabetes along with typically end-organ complications such as nephropathy, retinopathy, and peripheral neuropathy [33].

Idiopathic GP is described after all other metabolic, endocrine, neurologic, rheumatologic, paraneoplastic, postinfectious and surgical causes of GP have been excluded [29-31].

The most common symptoms of IGP are represented by nausea ( $>90 \%$ of patients), vomiting ( $84 \%$ of patients), and early satiety (60\% of patients). Rarely, postprandial fullness and upper abdominal pain appears, and weight loss, malnutrition, and dehydration appear only in severe cases [30-32]. In patients with mental disorders, such as depression or anxiety, higher anxiety/depression scores are correlated with the severity of the symptoms.

The dietary changes that can improve the symptomatology are: increasing the liquids ingestion rather than solids, consuming multiple low-fat small meals, and maintaining optimal glucose levels in diabetic patients. Male gender, gastrointestinal infections and the use of antidepressants, were proven to be predictors of symptom improvement by one study, and obesity, smoking, abdominal pain, gastroesophageal reflux disorder, severe depression were identified as predictors of no improvement [30-33].

A recent study demonstrated the importance of the genetic background in GP, showing that longer poly-GT that is repeating in the HMOX1 gene is more common in African Americans with GP and the symptomatology is more severe in subjects with longer alleles [34].

To diagnose GP one should assess gastric emptying. Among several techniques available, more frequently used are: scintigraphy, wireless motility capsule, and carbon 13 breath testing. Scintigraphy of a solid phase meal is still the standard [35]. A new method used to increase standardization is the use of a wireless motility capsule, called SmartPill which is measuring $\mathrm{pH}$, temperature, and pressure. It measures gastric emptying time calculating the duration from ingestion with a rise in $\mathrm{pH}$ from acidic antrum, untill it enters the alkaline medium of duodenum [35].

In clinical practice, the "Gastroparesis Symptom Cardinal Index" is used for a correct diagnosis and treatment. It uses a scale of six levels of severity from "very severe" to "none" and it evaluates: nausea, retching, vomiting, stomach fullness, not able to finish a normal-sized meal, feeling excessively full after meals, loss of appetite, bloating, stomach or belly visibly larger [33-35].

Prokinetic medications (metoclopramide, erythromycin) and antiemetic medications are usually used as treatment. Metoclopramide can cause extrapyramidal symptoms and should be administrated with caution to patients with anxiety or depression [36-38]. Furthermore, mirtazapine and other antidepressants may be an option by enhancing receptive relation and alleviating distress associated with gastroparesis [35-38]. Hypnotherapy is useful as reported by a recent systematic review [39].

\section{Gastroparesis-like syndrome}

Patients with GP with similar symptomatology but with normal gastric scintigraphy and non-delayed gastric emptying are defined as suffering from GLS. 
There is still debate over the definition and the differences between GLS and IGP. Gastric empting as diagnosed by nuclear scintigraphy may modify its clinical outcome in time and is considerable related to modifications by systemic processes (glycemic values, weight loss, etc) and medications [40].

Moreover, IGP and GLS patients included in studies are frequently heterogeneous, with similar symptoms, comorbidities, and response to treatment, complicating the protocol and the scientific outcome. Nevertheless, in the last decade, GLS is beginning to be recognized as a distinct pathological process.

The differential diagnosis between GLS and postprandial distress syndrome is made using the Rome IV Criteria and must include one or both of the following at least 3 days per week: 1) bothersome postprandial fullness (ie, severe enough to impact on usual activities);2) bothersome early satiation (ie, severe enough to prevent finishing a regular-size meal); 3) no evidence of organic, systemic, or metabolic disease that is likely to explain the symptoms on routine investigations (including at upper endoscopy); Criteria must be fulfilled for the last 3 months with symptom onset and at least 6 months before diagnosis [41].

The chemoreceptor trigger zone in the area postrema plays an essential role in the physiopathological process of emesis and is one of four principal areas that can induce emesis. Abdominal vagal afferents that identify potentially emetogenic substances in the lumen also terminate in this zone. Results from a wide variety of experiments show that the nucleus tractus solitarius connects to the emetic central pattern generator which is located in the retrofacial nucleus of the reticular formation playing a key role in the progression of various stimuli that will finally have the effect of emesis induction $[37,38,40,41]$. It controls respiratory groups that coordinate the muscular movements involved in retching and vomiting. There is no isolated emesis center but fragments of unorganized neurons in the medulla that can be activated by vestibular, cortical, gastro-intestinal and metabolic factors.

Studies have demonstrated that significantly more fibrosis is present in the pylorus in GP patients, compared with patients with GLS, that demonstrates that fibrosis is a main pathogenic factor in the process of delayed gastric emptying in GP patients [40]. Furthermore, in a retrospective analysis, pyloroplasty in GP patients was associated with improvement in symptoms and improved gastric emptying in $83 \%$ of patients [42].

A recent study performed by Bashashati et al. [43] compared the histopathological features of antral and pyloric smooth muscle tissue in GP and GP-like syndrome. The result was that 17 out of 24 (70.8\%) GP patients with pyloric biopsies had concomitant pyloric interstitial cells of Cajal (ICC) loss and severe fibrosis, while only one GLS patient had ICC loss [43]. Moreover, GP patients had a greater baseline total symptom score (TSS) compared to GP-like patients [43, 44]]. These pathological findings clearly explain the pyloric dysfunction and delayed gastric emptying in GP.

Gastric electrical stimulation (GES) is a new form of therapy for both GP and GLS and is recommended for patients with refractory emesis at pharmacological agents. While the efficacy and benefits of GES in gastroparesis is extensively documented, the role of GES in GLS is not precisely reviewed.
A study performed by Singh et al. [46] analyzed the effects of temporary gastric electrical stimulation (tGES) in patients with GLS. Total symptom score and scintigraphic parameters were also analyzed. 551 patients suffering from symptoms of gastroparesis (emesis, nausea, bloating/distension, anorexia/ early satiety, and abdominal pain) with negative endoscopy underwent gastric scintigraphy [46]. The following parameters were recorded: solid radio-nuclide gastric emptying, area under the gastric emptying curve and the total gastric emptying test. Patients were classified in three cathegories: delayed gastric emptying, normal gastric emptying, and rapid gastric emptying. Finally, from 551 patients in the larger cohort, 379 had implantation of tGES, symptoms and gastric emptying was compared pre and post tGES implantation $[45,46]$. The results were that after tGES, the gastric retention after 2 hours decreased $(p<0.01)$ for the delayed patients and increased $(p<0.001)$ for normal and rapid patients [46]. Considerable improvement in vomiting, nausea, and total symptom scores in all 3 subgroups, was demonstrated $(\mathrm{p}<0.001)$. Considering the recent data provided by studies of the neurophysiological mechanisms and pathogenesis of GP and GLS, the main recommendation for a research project for the future will be to determine which patients would benefit from the placement of a permanent gastric stimulator, which patients require surgery and which patients should be limited to pharmacological therapy.

\section{DISCUSSION}

Emesis is a frequent symptom in clinical medicine. However functional vomiting, associated with nausea, is difficult to diagnose and frustrating to treat. No therapeutically guidelines are available on CNVS, nor for GLS. There are guidelines for vomiting after chemotherapy of after surgery [47, 48].

Given the need of practitioners to be familiar with the entities of GBI presenting emesis, we reviewed the pertinent literature. The present review has identified articles, most of them prospective studies, about emesis in BGID during natural evolution or under therapy.

The pathophysiology behind emesis in DBGI is complex, with multiple and complex variables involved. Studies concentrating on histological changes in DBGI modify the basis of functional disorders paradigm and make more difficult the classification process of gastrointestinal disorders in organic and functional $[35,37,43]$. Furthermore, convincing data provided by the detailed analysis of genetic background of brain-gut disorders are changing the perspective of the psychosocial parameters as key determinants in the development long-term evolution of DBGI.

It is becoming obvious that precise mechanisms responsible for the development of different DBGI have to be further analyzed and compared in order to develop new pharmacological agents concentrating on the regulation of gastrointestinal motility.

Convincing evidence for the pathogenic mechanism implicated in the development of DBGI manifested with emesis is lacking. To date, IGP and CVS are the most frequently studied brain-gut disorders with emesis as the main symptom. Studies on CNVS and GLS, including case reports, are very limited. 
Our review is limited by the small number of patients included in the studies.

\section{CONCLUSIONS}

Emesis is the Cinderella of DBGI. Contradictory results about the implication of the genetic background and the phenotypic parameters in its occurrence reflect the missing knowledge of its pathogenesis. The management of functional emesis may prove difficult at times.

Conflicts of interest: None to declare.

Authors' contributions: S.L.P. searched the literature, drafted and wrote the manuscript. G.I.G. literature search; L.D. suggested the methodology analyzed the results, and revised the manuscript; G.C.critically revised the manuscript; D.L.D. supervised the work and substantial contributions to the writing. All authors approved the final version of the manuscript.

\section{REFERENCES}

1. Morra ME, Elshafay A, Kansakar AR, et al. Definition of "persistent vomiting" in current medical literature: A systematic review. Medicine (Baltimore) 2017;96:e8025. doi:10.1097/MD.0000000000008025

2. Burke RE. Sir Charles Sherrington's the integrative action of the nervous system: a centenary appreciation. Brain 2007;130:887-894. doi:10.1093/ brain/awm022

3. Drossman DA, Hasler WL. Rome IV-Functional GI Disorders: Disorders of Gut-Brain Interaction. Gastroenterology 2016;150:12571261. doi:10.1053/j.gastro.2016.03.035

4. Britt H, Fahridin S. Presentations of nausea and vomiting. Aust Fam Physician 2007;36:682-683.

5. Frese T, Klauss S, Herrmann K, Sandholzer H. Nausea and vomiting as the reasons for encounter. J Clin Med Res 2011;3:23-29. doi:10.4021/ jocmr410w

6. Metz A, Hebbard G. Nausea and vomiting in adults--a diagnostic approach. Aust Fam Physician 2007;36:688-692.

7. Quigley EM, Hasler WL, Parkham HP. AGA technical review on nausea and vomiting. Gastroenterology 2001;120:263-286. doi:10.1053/ gast.2001.20516

8. Sharkey KA, Wiley JW. The Role of the Endocannabinoid System in the Brain-Gut Axis. Gastroenterology 2016;151:252-266. doi:10.1053/j. gastro.2016.04.015

9. Aziz I, Palsson OS, Whitehead WE, Sperber AD, Simrén M, Törnblom H. Epidemiology, Clinical Characteristics, and Associations for Rome IV Functional Nausea and Vomiting Disorders in Adults. Clin Gastroenterol Hepatol 2019;17:878-886. doi:10.1016/j.cgh.2018.05.020

10. Drossman DA, Chang L, Chey WD, et al. (eds.). Rome IV-Functional GI Disorders: Disorders of Gut-Brain Interaction. Fourth Edition, Vol 2. Rome Foundation, 2016:943-944.

11. Li BU, Misiewicz L. Cyclic Vomiting Syndrome: a brain-gut disorder Gastroenterol Clin N Am 2003;32:997-1019. doi:10.1016/S0889. 8553(03)00045-1

12. Zeevenhooven J, Koppen IJ, Benninga MA. The New Rome IV Criteria for Functional Gastrointestinal Disorders in Infants and Toddlers. Pediatr Gastroenterol Hepatol Nutr 2017;20:1-13. doi:10.5223/ pghn.2017.20.1.1
13. Cullen KJ, MacDonald WB. The periodic syndrome: its nature and prevalence. Med J Aust 1963;50:167-173.

14. Venkatesan T, Zaki EA, Kumar N, et al. Quantitative pedigree analysis and mitochondrial DNA sequence variants in adults with cyclic vomiting syndrome. BMC Gastroenterol 2014;14:181. doi:10.1186/1471230X-14-181

15. Zaki EA, Freilinger T, Klopstock T, et al. Two common mitochondria DNA polymorphisms are highly associated with migraine headache and cyclic vomiting syndrome. Cephalalgia 2009;29:719-728. doi:10.1111/ j.1468-2982.2008.01793.x

16. Boles RG, Zaki EA, Lavenbarg T, et al. Are pediatric and adult-onset cyclic vomiting syndrome (CVS) biologically different conditions? Relationship of adult-onset CVS with the migraine and pediatric CVS-associated common mtDNA polymorphisms 16519T and 3010A Neurogastroenterol Motil 2009;21:936-e72. doi:10.1111/j.13652982.2009.01305.x

17. Hikita T, Kodama H, Ogita K, Kaneko S, Nakamoto N, Mimaki M. Cyclic Vomiting Syndrome in Infants and Children: A Clinical Follow-Up Study. Pediatr Neurol 2016;57:29-33. doi:10.1016/j. pediatrneurol.2016.01.001

18. Fleisher DR, Gornowicz B, Adams K, Burch R, Feldman EJ. Cyclic Vomiting Syndrome in 41 adults: the illness, the patients, and problems of management. BMC Med 2005;3:20. doi:10.1186/1741-7015-3-20

19. Fleisher DR. The cyclic vomiting syndrome described. J Pediatr Gastroenterol Nutr 1995;21 Suppl 1:S1-S5. doi:10.1097/00005176199501001-00003

20. Lapoint J, Meyer S, Yu CK, et al. Cannabinoid hyperemesis syndrome: public health implications and a novel model treatment guideline. West J Emerg Med 2018;19:380-386. doi:10.5811/westjem.2017.11.36368

21. Kim HS, Monte AA. Colorado cannabis legalization and its effect on emergency care. Ann Emerg Med 2016;68:71-75. doi:10.1016/j. annemergmed.2016.01.004

22. Kim HS, Anderson JD, Saghafi O, Heard KJ, Monte AA. Cyclic vomiting presentations following marijuana liberalization in Colorado. Acad Emerg Med 2015;22:694-699. doi:10.1111/acem.12655

23. Richards JR, Gordon BK, Danielson AR, Moulin AK. Pharmacologic treatment of cannabinoid hyperemesis syndrome: a systematic review. Pharmacotherapy 2017;37:725-734. doi:10.1002/phar.1931

24. Perrotta G, Miller J, Stevens T, et al. Cannabinoid hyperemesis: relevance to emergency medicine (abstract 542). Society for Academic Emergency Medicine Annual Meeting. 2012;19:S286-S287.

25. Chang YH, Windish DM. Cannabinoid hyperemesis relieved by compulsive bathing. Mayo Clin Proc 2009;84:76-78.

26. Patterson DA, Smith E, Monahan M, et al. Cannabinoid hyperemesis and compulsive bathing: a case series and paradoxical pathophysiological explanation. J Am Board Fam Med 2010;23:790-793. doi:10.3122/ jabfm.2010.06.100117

27. Sorensen CJ, DeSanto K, Borgelt L, Phillips KT, Monte AA. Cannabinoid Hyperemesis Syndrome: Diagnosis, Pathophysiology, and Treatment-a Systematic Review. J Med Toxicol 2017;13:71-87. doi:10.1007/s13181016-0595-Z

28. Darmani NA. The cannabinoid CB1 receptor antagonist SR 141716A reverses the antiemetic and motor depressant actions of WIN 55, 212-2. Eur J Pharmacol 2001;430:49-58. doi:10.1016/S0014-2999(01)01355-3

29. Fosso CL, Quigley EMM. A Critical Review of the Current Clinical Landscape of Gastroparesis. Gastroenterol Hepatol (N Y) 2018;14:140145.

30. Soykan I, Sivri B, Sarosiek I, Kiernan B, McCallum RW. Demography, clinical characteristics, psychological and abuse profiles, treatment, 
and long-term follow-up of patients with gastroparesis. Dig Dis Sci 1998;43:2398-2404. doi:10.1023/a:1026665728213

31. Parkman HP. Idiopathic gastroparesis. Gastroenterol Clin North Am 2015;44:59-68. doi:10.1016/j.gtc.2014.11.015

32. Nassara Y, Richter S. Gastroparesis in Non-Diabetics: Associated Conditions and Possible Risk Factors. Gastroenterology Res 2018;11:340-345. doi:10.14740/gr1060w

33. Munteanu MF, Gligor R, Crîsnic I, Costache CA, Colosi IA. Antimicrobial activity of Melampyrum cristatum, Melampyrum bihariense and Melampyrum arvense tinctures. Afr J Pharm Pharmacol 2012;6:2808-2812.doi:10.5897/AJPP12.404

34. Gibbons SJ, Grover M, Choi KM, et al. Repeat polymorphisms in the Homo sapiens heme oxygenase-1 gene in diabetic and idiopathic gastroparesis. PLoS One 2017;12:e0187772. doi:10.1371/journal.pone.0187772

35. Liu N, Abell T. Gastroparesis Updates on Pathogenesis and Management. Gut Liver 2017;11:579-589. doi:10.5009/gnl16336

36. Navas CM, Patel NK, Lacy BE. Gastroparesis: Medical and Therapeutic Advances. Dig Dis Sci 2017;62:2231-2240. doi:10.1007/s10620-017-4679-7

37. Kim BJ, Kuo B. Gastroparesis and Functional Dyspepsia: A Blurring Distinction of Pathophysiology and Treatment. J Neurogastroenterol Motil 2019;25:27-35. doi:10.5056/jnm18162

38. Nassar Y, Richter S. Gastroparesis in Non-Diabetics: Associated Conditions and Possible Risk Factors. Gastroenterology Res 2018;11:340-345. doi:10.14740/gr1060w

39. Stefan-Lucian P, Giuseppe C, Liliana D, Dan Lucian D. The Efficacy of Hypnotherapy in the Treatment of Functional Dyspepsia. Am J Ther 2019 Jul 9. doi:10.1097/MJT.0000000000001033

40. Gotfried J, Schey R. Understanding the Differences Between Gastroparesis and Gastroparesis-Like Syndrome: Filling a Gaping
Hole? Dig Dis Sci 2017;62:2615-2617. doi:10.1007/s10620-0174702-z

41. Suzuki H. The Application of the Rome IV Criteria to Functional Esophagogastroduodenal Disorders in Asia. J Neurogastroenterol Motil 2017;23:325-333.

42. Hibbard ML, Dunst CM, Swanström LL. Laparoscopic and endoscopic pyloroplasty for gastroparesis results in sustained symptom improvement. J Gastrointest Surg. 2011;15:1513-1519. doi:10.5056/ jnm17018

43. Bashashati M, Moraveji S, Torabi A, et al. Pathological Findings of the Antral and Pyloric Smooth Muscle in Patients with Gastroparesis-Like Syndrome Compared to Gastroparesis: Similarities and Differences. Dig Dis Sci 2017;62:2828-2833. doi:10.1007/s10620-017-4629-4

44. Bashashati M, McCallum RW. Motility: Is 'ICC-opathy' present in gastroparesis-like syndrome? Nat Rev Gastroenterol Hepatol 2015;12:375-376. doi:10.1038/nrgastro.2015.96

45. Wendorf G, Mehta M, Stocker A, Smith J, Abell T. Endoscopic aspects of temporary gastric electrical stimulator lead placement in patients with gastroparesis and gastroparesis-like syndromes. VideoGIE 2018;3:112. doi:10.1016/j.vgie.2018.01.007

46. Singh S, McCrary J, Kedar A, et al. Temporary endoscopic stimulation in gastroparesis-like syndrome. J Neurogastroenterol Motil 2015;21:520527. doi:10.5056/jnm15046

47. Gan TJ, Diemunsch P, Habib AS, et al. Consensus guidelines for the management of postoperative nausea and vomiting. Anesth Analg 2014;118:85-113. doi:10.1213/ANE.0000000000000002

48. Navari RM. Management of chemotherapy-induced nausea and vomiting in pediatric patients. Pediatric Drugs 2017;19:213-222. doi:10.1007/s40272-017-0228-2 